\title{
A Sutileza dos Quatérnions no Movimento de Rotação de Corpos Rígidos
}

\author{
The subtlety of the quaternions on the rigid body rotations
}

\author{
Andreyson Bicudo Jambersi*, Samuel da Silva ${ }^{\dagger}$
}

Departamento de Engenharia Mecânica, Faculdade de Engenharia de Ilha Solteira, Universidade Estadual Paulista Unesp, Ilha Solteira, SP, Brasil

Recebido em 18 de janeiro de 2016. Revisado em 19 de janeiro de 2016. Aceito em 30 de janeiro de 2016

A parametrização da velocidade angular instantânea de um corpo rígido é usualmente descrita nos cursos de mecânica clássica com o auxílio dos ângulos de Euler. No entanto, para evitar problemas de singularidade na integração numérica das equações de movimento de rotação, outras formas de parametrização são mais interessantes. Dentre as diversas opções, os quatérnions são uma escolha bastante popular e com forte apelo prático na parametrização e integração das equações de Euler. Neste sentido, o presente artigo contribui com uma apresentação didática e comparação da integração numérica das equações de Euler para descrição do movimento de um giroscópio com a cinemática inversa solucionada via ângulos de Euler e por quatérnions, que não são comumente descritas em livros didáticos clássicos em um primeiro curso de mecânica. Busca-se mostrar que além deste trunfo numérico, a integração das equações de movimento com parametrização via quatérnions também pode ser usada com um fim didático mais nobre: introduzir e motivar os estudantes de um curso de mecânica, tanto físicos como engenheiros, no uso de outros sistemas algébricos mais avançados, como a Álgebra Geométrica (Álgebra de Clifford).

Palavras-chave: Rotações. Giroscópio. Orientação no espaço. Singularidades. Ângulos de Euler. Quatérnions. Álgebra geométrica.

The angular velocity of a rigid body is usually described in classical mechanics courses by using Euler angles. However, in order to avoid singularity problems on the numerical integration of the motion equations, another ways are more interesting. Among them, the quaternions are a very popular choice and with a strong practical appeal on the parameterization and integration of the Euler's equations. In this sense, this paper proposes a comparison between the numerical integration of the Euler equations of a gyroscope with the inverse kinematics solved through Euler angles and by quaternions, that are not commonly described in classical mechanics textbooks on a first mechanical course. The goal is to show that apart the numerical asset, the integration of the motion equations with the quaternions parameterization also can be used with a noblest didactical way: to introduce and motivate the students with the use of others more advanced algebraic systems, as Geometric Algebra (Clifford's Algebra).

Keywords: Gyroscope, Quaternions, Euler Angles, Geometric Algebra, Rigid Body Rotation in Space.

\section{Introdução}

A parametrização de rotações finitas de um corpo rígido no Espaço Euclidiano foi descrita magistralmente por Euler em 1775 a partir do uso de três rotações sequenciais. Este resultado foi um corolário obtido a partir de seu teorema, conhecido hoje como

*Endereço de correspondência: andreysonj@gmail.com.br.

${ }^{\dagger}$ Endereço de correspondência: samuel@dem.feis.unesp.br.
Teorema de Rotação de Euler, que demonstrou que qualquer rotação de um corpo com um ponto fixo pode ser descrita por uma rotação própria em torno de um eixo de rotação, conhecido como Eixo de Euler, solidário ao giro do próprio corpo [1,2].

Até os dias atuais esta é uma das formas mais populares para representar a orientação de um corpo rígido no espaço. Os três ângulos de Euler parametrizam três rotações sequenciais [3] e são bastante 
visuais e claros, sendo utilizados para girar um corpo no espaço a partir de uma matriz de rotação com nove elementos, conhecidos como cossenos diretores. Porém, uma vez que o produto de matrizes não é, por via de regra, comutativo, a escolha e ordem destas rotações sequenciais deve ser definida a priori em função do tipo de problema. Em especial, um cuidado adicional deve ser levado em conta no momento desta escolha, pois as velocidades angulares instantâneas também são descritas por estes ângulos e suas respectivas taxas de variação. Um dos graves inconvenientes do uso dos ângulos de Euler para descrição destas velocidades é quando realizase algum cálculo envolvendo cinemática inversa, ou seja, quando a partir do conhecimento das velocidades angulares instantâneas, deseja-se integrar numericamente as equações para obter os ângulos que fornecem a orientação do corpo no espaço. Em algumas situações, existe uma clara singularidade que inviabiliza este cálculo de maneira direta 3 . Esse problema será discutido em mais detalhes na seção 4 deste artigo.

Felizmente, existem outras maneiras de parametrização que podem ser usadas com algumas vantagens frente ao uso dos ângulos de Euler [3]. Dentre estas formas, podem ser citados os parâmetros de Cayley-Klein [4], parâmetros de Rodrigues e Rodrigues Modificado [5, 6], vetor conforme [7] e, ainda, os quatérnions [8. Uma comparação das vantagens e desvantagens de algumas formas de parametrização pode ser encontrada em Trindade e Sampaio [7. Entretanto estas parametrizações de rotação não são discutidas em detalhes na maioria dos cursos de mecânica e são apresentadas em livros didáticos de mecânica clássica apenas de forma superficial 912 .

Dentre todas estas formas de parametrização, os quatérnions são um tanto quanto especiais, principalmente devido a sua origem histórica. William Rowan Hamilton, em meados do século XIX, sabia que multiplicação de números complexos eram isomorfos de rotação no $\mathbb{R}^{2}$ e buscou de forma persistente estender isto para rotações no $\mathbb{R}^{3} 13$. Suas primeiras tentativas envolveram utilizar elementos no $\mathbb{R}^{3}$ para operar rotações no $\mathbb{R}^{3}$, mas não obteve sucesso em obter uma álgebra fechada. Somente em 1843, Hamilton constatou que precisaria operar elementos no $\mathbb{R}^{4}$ para rotacionar elementos no $\mathbb{R}^{3}$. Este novo sistema numérico foi chamado por ele de quatérnion, sendo representado hoje por $\mathbb{H}$ e com uma propriedade bem interessante: quatérnions podem ser multiplica- dos, ao contrário de vetores ${ }^{1}$. Os quatérnions foram a primeira álgebra não-comutativa da história e Hamilton ficou bastante empolgado com o leque de possíveis aplicações em física e engenharia. Tanto que passou o resto de sua vida escrevendo e divulgando sobre os quatérnions, a que ele se referia como uma linguagem matemática universal para descrever fenômenos físicos diversos [14,15]. Infelizmente, no final do século XIX, o americano Gibbs e o inglês Heaviside popularizaram uma notação e "álgebra" de uma vertente, podendo ser entendida hoje como uma "versão simplificada" de quatérnions, separando o produto de dois quatérnions em duas operações separadas conhecidas hoje como produto vetorial, com o símbolo $\times$; e produto escalar, com o símbolo ·, na notação de Gibbs 16,17. Apesar de debates ferrenhos na época, a álgebra de quatérnions foi deixada no segundo plano e a "álgebra vetorial" de Gibbs, mesmo que com certos problemas e limitações, foi popularizada rapidamente, em especial devido a inúmeros livros didáticos escritos no início do século XX.

Apesar disto, na segunda metade do século XX, os quatérnions começaram a ser "redescobertos" e aplicados de forma maciça, especialmente em problemas de cinemática inversa em robótica e engenharia aeroespacial [18 20, visão computacional 21,22, biologia molecular [23 25], mecânica dos fluídos [26 28], mecânica quântica [29], entre inúmeras outras áreas. Porém, em grande parte destes artigos e trabalhos, em especial os de cunho técnico e de aplicações, os quatérnions são descritos como uma mera ferramenta matemática, ou método, usados para facilitar a integração numérica e evitar singularidades no problema de cinemática inversa. Carrera 30] afirma que os valores correspondentes das componentes do quatérnion não correspondem a nenhum significado físico, enquanto Oliveira [31] apresenta o uso de quatérnions como uma vantagem de cunho apenas prático na determinação da orientação de um veículo, por meio de um sistema de referência solidário ao corpo, cujo único objetivo é evitar singularidades. Alega-se, ainda, que pouco importa a facilidade em extrair algum significado físico dos quatérnions, já que eles podem ser facilmente convertidos para ângulos de Euler e que, estes sim, possuem a vantagem de permitirem o imediato entendi-

\footnotetext{
${ }^{1}$ Um objeto matemático corriqueiro hoje para descrever grandezas físicas, mas que em meados do século XIX não tinha a definição que temos hoje.
} 
mento físico. Altmann [32], por sua vez, apresenta os quatérnions como geradores de rotações no espaço. Lima e Mello 33 tratam os quatérnions como um método de interpretação não tão natural, destacando também a sua dificuldade de interpretação. Outros autores, por outro lado, alegam que a representação de rotações por quatérnions, quando comparada à parametrização por ângulos de Euler, tem a vantagem de ser livre da complexidade analítica que envolve os ângulos de Euler [34]. Porém, da mesma forma que números complexos, entidades às quais os estudantes já estão, em muitos casos, habituados e que são intuitivas quando tratam-se rotações no plano, a representação de quatérnions puros no espaço tridimensional também o é, já que por si só fornece uma visualização das trajetórias que são muito mais expressivas do que a decomposição em termos de três ângulos de Euler relacionados a um sistema inercial, fornecendo uma representação matemática e espacial muito simples de rotações. Essa facilidade de visualização, quando combinada com a simplicidade de implementação computacional e ao número menor de operações matemáticas necessárias para realizar rotações, não só traz uma redução no tempo de processamento ao representar trajetórias, como é facilmente compreendida uma vez entendido o formalismo matemático por trás dos quatérnions. Outra questão é que, quando optar-se por expressar os resultados dessas rotações em termos de grandezas angulares, pode-se calculá-las facilmente a partir dos quatérnions.

No entanto, o que alguns destes autores que usam quatérnions não ressaltam é que o uso de quatérnions para parametrizar rotações tem uma função e significado muito mais profundo do que apenas servir de um artifício ou método numérico para evitar singularidades. Neste sentido, este artigo tem como meta descrever esta ideia a partir de um problema concreto envolvendo o estudo clássico de orientação de um giroscópio. A integração numérica das equações de movimento que descrevem o movimento de orientação de um giroscópio pode ter a velocidade angular parametrizada com ângulos de Euler e quatérnions para condição livre e com aplicação de torques externos, com ou sem problema de singularidade numérica dependendo das condições iniciais. A partir este problema, o presente artigo discute como é possível estender e apresentar os quatérnions como uma classe algébrica particular de um sistema mais avançado: a álgebra geométrica (álgebra de
Clifford). Assim, em um curso de mecânica clássica é possível introduzir a álgebra geométrica como uma classe especial apresentando os quatérnions como uma álgebra fechada com operação de multiplicação não-comutativa entre os seus elementos que, além de um enorme apelo prático na solução de problemas relevantes e de interesse diverso, serve como porta de entrada para a Álgebra Geométrica.

O artigo está organizado em três partes principais. Inicialmente apresenta-se uma introdução à álgebra dos quatérnions, mostrando as suas principais propriedades e como realizar rotações com estes elementos. Na sequência, se revisa as equações de Euler para orientação de um giroscópio no espaço, seguido do problema da cinemática inversa, solucionado via ângulos de Euler e quatérnions. Em seguida, discute-se como entender os quatérnions como uma álgebra de Clifford. Por fim, algumas considerações finais são pontuadas ao final do texto.

\section{A Álgebra dos Quatérnions}

Os quatérnions consistem em uma generalização dos números complexos para quatro dimensões e são compostos por uma parte escalar $q_{0}$ e uma parte vetorial q. Pode-se tratar os números reais como números hipercomplexos de dimensão 1 e os números complexos comuns como hipercomplexos de dimensão 2. Nesses casos, relações de comutatividade tanto para soma quanto para multiplicação são sempre válidas. Por outro lado, para qualquer hipercomplexo de dimensão superior a 2, essas relações não são necessariamente válidas. Um caso especial são os hipercomplexos de dimensão 4, conhecidos como quatérnions [8].

A fig. 11 descreve uma regra mnemônica para representar a não-comutatividade das multiplicações de quatérnions, onde a multiplicação de dois termos em sentido horário fornece como resultado o terceiro termo com sinal positivo. Por exemplo, se for multiplicados ij tem-se como resultado $k$, já a multiplicação de dois termos subsequentes em sentido anti-horário também fornece o terceiro termo, porém, com sinal negativo, ou seja quando for multiplicado $j i$, tem-se como resultado $-k$.

Deste modo, observa-se que os componentes unitários $i, j, k$ do quatérnion respeitam as relações fundamentais: 


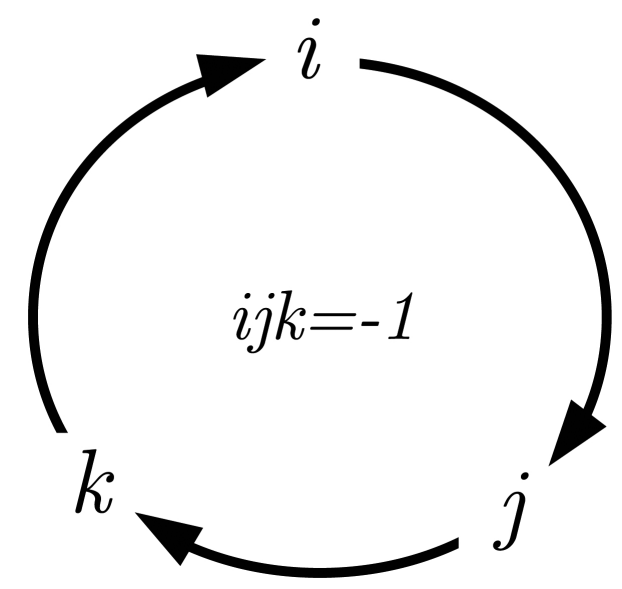

Figura 1: Representação gráfica da regra mnemônica de multiplicações de quatérnions.

$$
\begin{array}{r}
i^{2}=j^{2}=k^{2}=i j k=-1 \\
i j=k=-j i \\
j k=i=-k j \\
k i=j=-i k
\end{array}
$$

representado por:

$$
\hat{q}=q_{0}+q_{1} i+q_{2} j+q_{3} k
$$

ou por:

$$
\hat{q}=\left\{\begin{array}{llll}
q_{0} & q_{1} & q_{2} & q_{3}
\end{array}\right\}^{T}
$$

ou ainda, por:

$$
\hat{q}=\left\{\begin{array}{l}
q_{0} \\
\mathbf{q}
\end{array}\right\}
$$

sendo

$$
\mathbf{q}=\left\{\begin{array}{lll}
q_{1} & q_{2} & q_{3}
\end{array}\right\}^{T}
$$

Assim, os quatérnions são compostos por quatro componentes ortogonais. Quando a parte escalar $q_{0}$ do quatérnion é nula, tem-se um quatérnion puro, que pode, por exemplo, representar um vetor de três dimensões, do mesmo modo escalares podem ser tratados como quatérnions com parte vetorial $\mathbf{q}$ nula 35 .

Um quatérnion $\hat{q}$ possui um conjugado $\hat{q}^{*} \mathrm{e}$ módulo $|\hat{q}|$ tal que:

$$
\begin{gathered}
\hat{q}^{*}=q_{0}-\mathbf{q} \\
|\hat{q}|=\sqrt{q_{0}^{2}+q_{1}^{2}+q_{2}^{2}+q_{3}^{2}}
\end{gathered}
$$

Os quatérnions têm fechamento na soma, assim para $\forall \hat{q}, \hat{p} \in \mathbb{H}$, vale que $\hat{q}+\hat{p} \in \mathbb{H}$, onde $\mathbb{H}$ denota o conjunto dos quatérnions. Estes também apresentam fechamento na multiplicação por escalar, assim $\forall \alpha \epsilon$ $\mathbb{R}$ e $\forall \hat{q} \in \mathbb{H}$, vale $\alpha \hat{q} \in \mathbb{H}$. Também são válidos os seguintes axiomas, que mostram que os quatérnions definem um espaço vetorial:

- $\hat{q}+\hat{p}=\hat{p}+\hat{q}$

- $\hat{q}+\hat{0}=\hat{q}$

- $\alpha(\hat{q}+\hat{p})=\alpha \hat{q}+\alpha \hat{p}$

- $(\alpha+\beta) \hat{q}=\alpha \hat{q}+\beta \hat{q}$

- $1 \hat{q}=\hat{q}$

- $\hat{q}+(\hat{p}+\hat{w})=(\hat{q}+\hat{p})+\hat{w}$

- $\hat{q}-\hat{q}=\hat{0}$

- $\beta(\alpha \hat{q})=(\alpha \beta) \hat{q}$

Assim, o conjunto dos quatérnions é um isomorfo do $\mathbb{R}^{3}$ para quatérnions puros e é isomorfo do $\mathbb{R}^{4}$ para os demais casos.

Observa-se também que, por não satisfazer a comutatividade na multiplicação, o conjunto numérico $\mathbb{H}$ não forma um corpo. Já a inversa de um quatérnion é definida por:

$$
\hat{q}^{-1} \hat{q}=\hat{q} \hat{q}^{-1}=1
$$

Kuipers [8 mostra que se $\hat{q}$ for um quatérnion unitário, ou normalizado, a inversa do quatérnion é próprio complexo conjugado, de modo que:

$$
\hat{q}^{-1}=\hat{q}^{*}
$$

Interessante ressaltar que os quatérnions são excelentes operadores de rotação. Sejam $\forall$ dois vetores $\mathbf{w}, \mathbf{v} \in \mathbb{R}^{3}$ que podem ser descritos como quatérnions puros, de tal modo que $\hat{w}=0+\mathbf{w}$ e $\hat{v}=0+\mathbf{v} \in \mathbb{H}$, onde $\hat{w}$ é $\hat{v}$ após a operação de rotação. Pode-se definir um operador de rotações $\mathscr{L}_{q}$, associado com o quatérnion $\hat{q}$ e aplicado ao vetor $\mathbf{v}$, a partir de:

$$
\begin{array}{r}
\hat{w}=\mathscr{L}_{q}(\mathbf{v})=\hat{q} \mathbf{v} \hat{q}^{*}=\left(q_{0}^{2}-\left|\mathbf{q}^{2}\right|\right) \mathbf{v}+ \\
2(\mathbf{q} \cdot \mathbf{v}) \mathbf{q}+2 q_{0}(\mathbf{q} \times \mathbf{v})
\end{array}
$$

sendo $\mathscr{L}_{q}$ um operador linear representando geometricamente uma rotação de um ângulo $\alpha$ em torno de um vetor diretor $\mathbf{u}$ no $\mathbb{R}^{3}$, onde $\mathbf{u}$ fornece a orientação do eixo de rotação do Teorema de Rotação de Euler e $\alpha$ o ângulo de rotação em torno deste eixo. A representação em quatérnions é, então, fornecida por um quatérnion unitário $\hat{q}$ na forma:

$$
\hat{q}=\cos \left(\frac{\alpha}{2}\right)+\mathbf{u} \operatorname{sen}\left(\frac{\alpha}{2}\right)
$$


A operação de rotações com quatérnions pode ser ainda escrita na forma explícita $\mathbf{w}=\mathcal{Q} \mathbf{v}$, de modo que:

$$
\underbrace{\left\{\begin{array}{l}
w_{1} \\
w_{2} \\
w_{3}
\end{array}\right\}}_{\mathbf{w}}=\underbrace{\left[\begin{array}{lll}
2\left(q_{0}^{2}+q_{1}^{2}\right)-1 & 2\left(q_{1} q_{2}+q_{0} q_{3}\right) & 2\left(q_{1} q_{3}-q_{0} q_{2}\right) \\
2\left(q_{1} q_{2}-q_{0} q_{3}\right) & 2\left(q_{0}^{2}+q_{2}^{2}\right)-1 & 2\left(q_{2} q_{3}+q_{0} q_{1}\right) \\
2\left(q_{1} q_{3}+q_{0} q_{2}\right) & 2\left(q_{2} q_{3}-q_{0} q_{1}\right) & 2\left(q_{0}^{2}+q_{3}^{2}\right)-1
\end{array}\right]}_{\mathcal{Q}} \underbrace{\left\{\begin{array}{l}
v_{1} \\
v_{2} \\
v_{3}
\end{array}\right\}}_{\mathbf{v}}
$$

Essa representação permite a comparação da matriz de rotações composta por quatérnions com a matriz de rotações envolvendo funções trigonométricas dos ângulos de Euler. Essa relação entre as duas matrizes será realizada na seção 4 , nas equações (38), (39) e (40).

\section{Orientação de um Giroscópio}

Um giroscópio pode ser descrito por um disco com massa $m$ e raio $R$, posicionado em $O$ e girando em torno de seu eixo de simetria, conforme visto na fig. 2. O sistema inercial $\mathscr{I}$ é fixo em $O$, que corresponde ao centro de massa, com os eixos $(x, y, z)$ representado pela base ortonormal $\{\hat{i}, \hat{j}, \hat{k}\}$. O primeiro giro, de precessão, ocorre em torno de $z \equiv z_{1}$ com velocidade angular $\dot{\psi}$ e é solidário ao sistema móvel $\mathscr{B}_{1}$ descrito por $\left(x_{1}, y_{1}, z_{1}\right)$ representado pela base ortonormal $\left\{\hat{i}_{1}, \hat{j}_{1}, \hat{k}_{1}\right\}$. Já o segundo giro, de nutação, ocorre em torno de $y_{1} \equiv y_{2}$ com velocidade angular $\dot{\theta}$ e é solidário ao sistema móvel $\mathscr{B}_{2}$ descrito por $\left(x_{2}, y_{2}, z_{2}\right)$ com a base $\left\{\hat{i}_{2}, \hat{j}_{2}, \hat{k}_{2}\right\}$. Por fim o terceiro giro, de spin, ocorre em torno de $z_{2} \equiv z_{3}$ com velocidade angular $\dot{\phi}$ e é, por sua vez, solidário ao sistema móvel $\mathscr{B}_{3}$ descrito por $\left(x_{3}, y_{3}, z_{3}\right)$ com a base $\left\{\hat{i}_{3}, \hat{j}_{3}, \hat{k}_{3}\right\}$. Todos estes eixos e giros são descritos na fig. 2. O movimento da base $\mathscr{B}_{3}$ é solidário ao do corpo, e o tensor de inércia $\mathscr{B}_{3} \mathbb{I}_{O}$ é:

$$
\mathscr{B}_{3} \mathbb{I}_{O}=\left[\begin{array}{ccc}
I_{x_{3}} & 0 & 0 \\
0 & I_{y_{3}} & 0 \\
0 & 0 & I_{z_{3}}
\end{array}\right], \quad \text { sendo: }\left\{\begin{array}{r}
I_{x_{3}}=\frac{1}{2} m R^{2} \\
I_{y_{3}}=I_{z_{3}}=\frac{1}{4} m R^{2}
\end{array}\right.
$$

O tensor de inércia, calculado a partir dos valores de parâmetros de massa $m=2 \mathrm{~kg}$ e raio $R=0.075 \mathrm{~m}$ do giroscópio, é representado na base móvel $\mathscr{B}_{3}$ como:

$$
\mathscr{B}_{3} \mathbb{I}_{O}=\left[\begin{array}{ccc}
0.0056 & 0 & 0 \\
0 & 0.0028 & 0 \\
0 & 0 & 0.0028
\end{array}\right] \text { unidade: }\left[\mathrm{kg} \cdot \mathrm{m}^{2}\right]
$$

A matriz de rotação entre os sistemas $\mathscr{I}$ e $\mathscr{B}_{3}$ pode ser escrita como:

$$
\mathcal{R}(\psi, \theta, \phi)=\left[\begin{array}{ccc}
\cos \psi \cos \theta \cos \phi-\operatorname{sen} \psi \operatorname{sen} \phi & \cos \psi \operatorname{sen} \phi+\operatorname{sen} \psi \cos \theta \cos \phi & -\operatorname{sen} \theta \cos \phi \\
-(\cos \psi \cos \theta \operatorname{sen} \phi+\operatorname{sen} \psi \cos \phi) & \cos \psi \cos \phi-\operatorname{sen} \psi \cos \theta \operatorname{sen} \phi & \operatorname{sen} \theta \operatorname{sen} \phi \\
\cos \psi \operatorname{sen} \theta & \operatorname{sen} \psi \operatorname{sen} \theta & \cos \theta
\end{array}\right]
$$

Já a velocidade angular do corpo, em função dos ângulos de Euler e representada na base $\mathscr{B}_{3}$, é descrita por:

$$
\mathscr{B}_{3} \boldsymbol{\omega}=\left\{\begin{array}{c}
\omega_{x 3} \\
\omega_{y 3} \\
\omega_{z 3}
\end{array}\right\}=\left\{\begin{array}{c}
-\dot{\psi} \operatorname{sen} \theta \cos \phi+\dot{\theta} \operatorname{sen} \phi \\
\dot{\psi} \operatorname{sen} \theta \operatorname{sen} \phi+\dot{\theta} \cos \phi \\
\dot{\psi} \cos \theta+\dot{\phi}
\end{array}\right\}
$$

sendo $\omega_{x 3}, \omega_{y 3}$ e $\omega_{z 3}$ os componentes da velocidade angular do giroscópio nos eixos principais de inércia.

O momento angular do giroscópio representado no sistema móvel $\mathscr{B}_{3}$, pode ser descrito pela relação:

$$
\mathscr{B}_{3} \mathscr{H}=\mathscr{B}_{3} \mathbb{I}_{O \mathscr{B}_{3}} \omega
$$




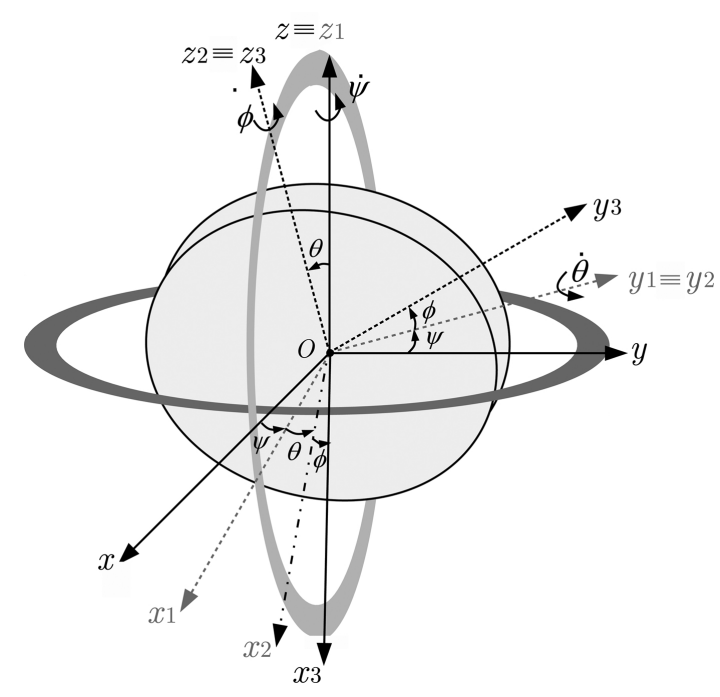

Figura 2: Desenho esquemático do giroscópio e seus sistemas de referência.

sendo $\mathscr{B}_{3} \mathscr{H}$ o momento angular do giroscópio.

As equações para descrever a orientação do giroscópio são obtidas a partir do cálculo da taxa de variação do momento angular, descrita por:

$$
\mathscr{B}_{3} \mathbf{M}=\left\{\begin{array}{l}
M_{x 3} \\
M_{y 3} \\
M_{z 3}
\end{array}\right\}=\frac{d}{d t}\left(\mathscr{B}_{3} \mathscr{H}\right)+\mathscr{B}_{3} \Omega \times_{\mathscr{B}_{3}} \mathscr{H}
$$

sendo $\mathscr{B}_{3} \boldsymbol{\Omega}=\mathscr{B}_{3} \boldsymbol{\omega}$ a velocidade angular do sistema de referência e $\mathscr{B}_{3} \mathbf{M}$ a soma dos torques externos, representados no sistema de referência móvel $\mathscr{B}_{3}$.

Calculando a derivada da amplitude do momento angular e a taxa de variação da direção do momento angular, obtém-se as equações de Euler de primeira ordem:

$$
\begin{aligned}
& \dot{\omega}_{x 3}=\frac{\left(I_{y 3}-I_{z 3}\right)}{I_{x 3}} \omega_{y 3} \omega_{z 3}+\frac{M_{x 3}}{I_{x 3}} \\
& \dot{\omega}_{y 3}=\frac{\left(I_{z 3}-I_{x 3}\right)}{I_{y 3}} \omega_{z 3} \omega_{x 3}+\frac{M_{y 3}}{I_{y 3}} \\
& \dot{\omega}_{z 3}=\frac{\left(I_{x 3}-I_{y 3}\right)}{I_{z 3}} \omega_{x 3} \omega_{y 3}+\frac{M_{z 3}}{I_{z 3}}
\end{aligned}
$$

que podem ser integradas numericamente para obtenção das velocidades instantâneas $\omega_{x 3}, \omega_{y 3}$ e $\omega_{z 3}$. Os ângulos de Euler $\psi, \theta$ e $\phi$ são obtidos pela integração inversa da equação 15 .

\section{Cinemática Inversa via Ângulos de Eu- ler versus Quatérnions}

O problema da cinemática inversa por meio dos ângulos de Euler consiste em relacionar as velo- cidades de precessão $\dot{\psi}$, nutação $\dot{\theta}$ e spin $\dot{\phi}$ com as velocidades angulares nas direções principais de inércia, explicitando $\dot{\psi}, \dot{\theta}$ e $\dot{\phi}$ a partir da inversa da eq. (15):

$$
\left\{\begin{array}{ccc}
\dot{\psi} & = & \frac{1}{\operatorname{sen} \theta}\left(\omega_{y 3} \operatorname{sen} \phi-\omega_{x 3} \cos \phi\right) \\
\dot{\theta} & = & \omega_{x 3} \operatorname{sen} \phi+\omega_{y 3} \cos \phi \\
\dot{\phi} & = & \omega_{z 3}-\dot{\psi} \cos \theta
\end{array}\right.
$$

Estas equações podem ser integradas numericamente. No entanto, para $\theta=n \pi$, com $|n|=0,2,4,6 \ldots$ tem-se que $\operatorname{sen} \theta \approx 0$, de modo que a eq. (19) pode apresentar singularidade. Este inconveniente de singularidade não ocorre utilizando os quatérnions para representar rotações. Para a solução de um problema deste tipo, inicialmente é necessário estimar os quatérnions correspondentes às condições iniciais do problema, o que pode ser feito com a eq. (11). No caso de rotações sequenciais, sejam $\hat{q}_{z 1}, \hat{q}_{y 2} \mathrm{e}$ $\hat{q}_{z 3}$ as rotações em torno dos eixos $z \equiv z_{1}, y_{1} \equiv y_{2} \mathrm{e}$ $z_{2} \equiv z_{3}$, respectivamente:

$$
\begin{aligned}
& \hat{q}_{z 1}=\cos \frac{\psi}{2}+k \operatorname{sen} \frac{\psi}{2} \\
& \hat{q}_{y 2}=\cos \frac{\theta}{2}+j \operatorname{sen} \frac{\theta}{2} \\
& \hat{q}_{z 3}=\cos \frac{\phi}{2}+k \operatorname{sen} \frac{\phi}{2}
\end{aligned}
$$

A rotação total, resultante das três rotações sequenciais, será:

$$
\hat{q}=\hat{q}_{z 3} \hat{q}_{y 2} \hat{q}_{z 1}=q_{0}+i q_{1}+j q_{2}+k q_{3}
$$

onde, aplicando as propriedades de multiplicação de quatérnions, pode-se obter as componentes $q_{i}^{\prime} s$ do quatérnion $\hat{q}$ :

$$
\begin{aligned}
& q_{0}=\cos \frac{\psi}{2} \cos \frac{\theta}{2} \cos \frac{\phi}{2}-\operatorname{sen} \frac{\psi}{2} \cos \frac{\theta}{2} \operatorname{sen} \frac{\phi}{2} \\
& q_{1}=\cos \frac{\psi}{2} \operatorname{sen} \frac{\theta}{2} \operatorname{sen} \frac{\phi}{2}-\operatorname{sen} \frac{\psi}{2} \operatorname{sen} \frac{\theta}{2} \cos \frac{\phi}{2} \\
& q_{2}=\cos \frac{\psi}{2} \operatorname{sen} \frac{\theta}{2} \cos \frac{\phi}{2}+\operatorname{sen} \frac{\psi}{2} \operatorname{sen} \frac{\theta}{2} \operatorname{sen} \frac{\phi}{2} \\
& q_{3}=\operatorname{sen} \frac{\psi}{2} \cos \frac{\theta}{2} \cos \frac{\phi}{2}+\cos \frac{\psi}{2} \cos \frac{\theta}{2} \operatorname{sen} \frac{\phi}{2}
\end{aligned}
$$

Calculados os termos $q_{0}, q_{1}, q_{2}$ e $q_{3}$, pode-se integrar também as equações de Euler, eq. (18), e relacionar a taxa de variação de um quatérnion $\hat{\dot{q}}$ com as velocidades angulares na direção principal, escritas na forma de quatérnions. Essa relação pode ser obtida a partir da ideia de que entre dois 
quatérnions unitários pode haver um terceiro que represente a transição entre eles [8. As formas de obtenção deste quatérnion são discutidas em 36. Este quatérnion de transição pode ser, então, escrito como:

$$
\hat{q}(t+\Delta t)=\hat{q}(t) \Delta \hat{r}(t)
$$

sendo:

$$
\Delta \hat{r}(t)=\cos \frac{\Delta \alpha}{2}+\mathbf{u}(t) \operatorname{sen} \frac{\Delta \alpha}{2}
$$

o incremento da transição entre os quatérnions. Seu ângulo de rotação é $\Delta \alpha$ e ocorre em torno de um eixo definido pelo vetor diretor $\mathbf{u}(t)$. Supondo que o incremento $\frac{\Delta \alpha}{2}$ é pequeno, assume-se a velocidade angular $\omega(t)$ como constante no intervalo de tempo $\Delta t$, e pode-se assumir $\cos \left(\frac{\Delta \alpha}{2}\right) \approx 1$ e sen $\left(\frac{\Delta \alpha}{2}\right) \approx \frac{\Delta \alpha}{2}$, de forma que a eq. 26 pode ser reescrita como:

$$
\Delta \hat{r}(t)=1+\mathbf{u}(t) \frac{\Delta \alpha}{2}
$$

substituindo este resultado na eq 25), obtém-se:

$$
\hat{q}(t+\Delta t)-\hat{q}(t)=\hat{q}(t) \mathbf{u}(t) \frac{\Delta \alpha}{2}
$$

se tratarmos o vetor $\mathbf{u}(t)$ como um quatérnion de parte escalar nula,

$$
\hat{q}(t+\Delta t)-\hat{q}(t)=\frac{\Delta \alpha}{2}\left\{\begin{array}{c}
0 \\
\mathbf{u}(t)
\end{array}\right\}
$$

e dividindo ambos os lados da eq. (28) por $\Delta t$ e aplicando o limite para $\Delta t \rightarrow 0$ :

$$
\begin{array}{r}
\frac{d \hat{q}(t)}{d t}=\lim _{\Delta t \rightarrow 0} \frac{\hat{q}(t+\Delta t)-\hat{q}(t)}{\Delta t}= \\
\lim _{\Delta t \rightarrow 0} \frac{1}{2} \hat{q}(t) \frac{\Delta \alpha}{\Delta t}\left\{\begin{array}{c}
0 \\
\mathbf{u}(t)
\end{array}\right\}
\end{array}
$$

e, sendo $\dot{\alpha}=\frac{\Delta \alpha}{\Delta t}$ a taxa de variação de $\alpha$ :

$$
\frac{d \hat{q}(t)}{d t}=\frac{1}{2} \hat{q}(t) \dot{\alpha}\left\{\begin{array}{c}
0 \\
\mathbf{u}(t)
\end{array}\right\}
$$

sendo a velocidade angular do quatérnion incremental $\Delta \hat{r}(t)$ :

$$
\boldsymbol{\omega}(t)=\dot{\alpha} \mathbf{u}(t)
$$

finalmente, obtém-se:

$$
\frac{d \hat{q}(t)}{d t}=\frac{1}{2} \hat{q}(t)\left\{\begin{array}{c}
0 \\
\omega(t)
\end{array}\right\}
$$

Onde a velocidade angular está descrita como um quatérnion de parte escalar nula $\hat{\omega}=\left\{\begin{array}{ll}0 & \boldsymbol{\omega}\end{array}\right\}^{T}$. A eq. (33) pode, então, ser reescrita como:

$$
\frac{d \hat{q}(t)}{d t}=\frac{1}{2} \hat{q}(t)\left\{\begin{array}{c}
0 \\
\omega_{x 3} \\
\omega_{y 3} \\
\omega_{z 3}
\end{array}\right\}
$$

ou, ainda, a partir da definição de produto entre dois quatérnions:

$$
\begin{aligned}
\left\{\begin{array}{l}
\dot{q}_{0} \\
\dot{q}_{1} \\
\dot{q}_{2} \\
\dot{q}_{3}
\end{array}\right\} & =\frac{1}{2}\left(q_{0}+\mathbf{q}\right)(0+\boldsymbol{\omega}) \\
& =q_{0} \cdot 0-\mathbf{q} \cdot \boldsymbol{\omega}+0 \cdot \mathbf{q}+q_{0} \boldsymbol{\omega}+\mathbf{q} \times \boldsymbol{\omega} \\
& =-\mathbf{q} \cdot \boldsymbol{\omega}+q_{0} \boldsymbol{\omega}+\mathbf{q} \times \boldsymbol{\omega}
\end{aligned}
$$

resolvendo as operações de produto escalar e produto vetorial descritas na eq. (35), obtém-se:

$$
\begin{gathered}
\dot{q}_{0}=-\frac{1}{2}\left(q_{1} \omega_{x 3}+q_{2} \omega_{y 3}+q_{3} \omega_{z 3}\right) \\
\dot{q}_{1}=\frac{1}{2}\left(q_{0} \omega_{x 3}+q_{3} \omega_{y 3}-q_{2} \omega_{z 3}\right) \\
\dot{q}_{2}=\frac{1}{2}\left(-q_{3} \omega_{x 3}+q_{0} \omega_{y 3}+q_{1} \omega_{z 3}\right) \\
\dot{q}_{3}=\frac{1}{2}\left(q_{2} \omega_{x 3}-q_{1} \omega_{y 3}+q_{0} \omega_{z 3}\right)
\end{gathered}
$$

que pode ser reescrita, na forma matricial, como:

$$
\left\{\begin{array}{c}
\dot{q}_{0} \\
\dot{q}_{1} \\
\dot{q}_{2} \\
\dot{q}_{3}
\end{array}\right\}=\frac{1}{2}\left[\begin{array}{cccc}
q_{0} & -q_{1} & -q_{2} & -q_{3} \\
q_{1} & q_{0} & -q_{3} & q_{2} \\
q_{2} & q_{3} & q_{0} & -q_{1} \\
q_{3} & -q_{2} & q_{1} & q_{0}
\end{array}\right]\left\{\begin{array}{c}
0 \\
\omega_{x 3} \\
\omega_{y 3} \\
\omega_{z 3}
\end{array}\right\}
$$

Em suma, para resolver o problema de cinemática inversa por quatérnions, deve-se integrar as equações de Euler, eq. (18), e substituir os valores encontrados para as velocidades angulares nas equações (37) e, em seguida, integrar as equações (37) para a obtenção dos quatérnions $\hat{q}(t)$ em cada instante de tempo. Caso opte-se por determinar os ângulos de Euler, pode-se relacionar a matriz de rotação $\mathcal{R}$, eq. (14), à forma explícita da operação de rotações com quatérnions $\mathcal{Q}$, visto na eq. (12). Igualandose as matrizes termo a termo, ou seja $\mathcal{Q}_{i j}=\mathcal{R}_{i j}$ e realizando-se algumas operações algébricas entre esses termos à fim de isolar os ângulos $\psi, \theta$ e $\phi$, obtém-se:

$$
\psi=\arctan \left(\frac{\mathcal{Q}(3,2)}{\mathcal{Q}(3,1)}\right)=\arctan \left(\frac{2\left(q_{2} q_{3}-q_{0} q_{1}\right)}{2\left(q_{1} q_{3}+q_{0} q_{2}\right)}\right)
$$




$$
\begin{gathered}
\theta=\operatorname{arcsen}(\mathcal{Q}(3,3))=\operatorname{arcsen}\left(2\left(q_{0}^{2}+q_{3}^{2}\right)-1\right) \\
\phi=\arctan \left(\frac{-\mathcal{Q}(2,3)}{\mathcal{Q}(1,3)}\right)=\arctan \left(\frac{-2\left(q_{2} q_{3}+q_{0} q_{1}\right)}{2\left(q_{1} q_{3}-q_{0} q_{2}\right)}\right)
\end{gathered}
$$

Neste ponto são realizadas as integrações numéricas das equações (18) e (19) para o problema da cinemática inversa por ângulos de Euler; e das equações (18) e (37) para o problema da cinemática inversa por quatérnions. Todas as integrações foram feitas utilizando o método de Runge-Kutta de $4^{\mathrm{O}}$ ordem, com 10000 amostras temporais e passo de integração $\Delta t=1.0 \times 10^{-3} \mathrm{~s}$. Foi assumido apenas o caso onde o sistema está livre da ação de momentos externos, com $\mathscr{B}_{3} \mathbf{M}=\mathbf{0}$ N.m.

A fig. 3 apresenta os ângulos de Euler obtidos para um caso onde não existia singularidade, de modo que a solução por meio da cinemática inversa por ângulos de Euler e por quatérnions apresentaram resultados idênticos entre si. A solução apresentada é aquela obtida por cinemática inversa via ângulos de Euler e optou-se por omitir a solução por quatérnions para evitar a repetição de resultados. Para esta situação, a velocidade de spin foi definida com valor muito superior às velocidades de precessão e nutação. Notase, portanto, um ligeiro aumento na velocidade de spin bem como do próprio ângulo de spin $\phi(t)$, enquanto os ângulos de precessão $\psi(t)$ e nutação $\theta(t)$ apresentaram comportamento de caráter oscilatório, com tendência ao aumento de suas amplitudes. Para o estudo realizado desprezou-se qualquer presença de atrito ou dissipação de energia.

A fig. 4 exibe os resultados para as velocidades angulares nas direções principais de inércia, nota-se que na fig. 4(a) que $\omega_{x 3}(t)$ é constante ao longo do tempo, o que ocorre pois, se observamos a equação de Euler, eq. (18), da qual da integração resultam $\omega_{x 3}(t), \omega_{y 3}(t)$ e $\omega_{z 3}(t)$, se $M_{x 3}=0 \mathrm{Nm}$ e $I_{y 3}=I_{z 3}$, obtém-se que $\dot{\omega}_{x 3}=0$, cujo resultado da integração fornece um valor constante para $\omega_{x 3}(t)$.

A fig. 5, apresenta as componentes $q_{0}, q_{1}, q_{2}$ e $q_{3}$ do quatérnion $\hat{q}$ calculado no problema de cinemática inversa utilizando-se os quatérnions. $\mathrm{O}$ módulo de $\hat{q}$, obtido a partir da eq. (7) é igual a 1 para qualquer instante de tempo, garantindo que a operação realizada é apenas de rotação.

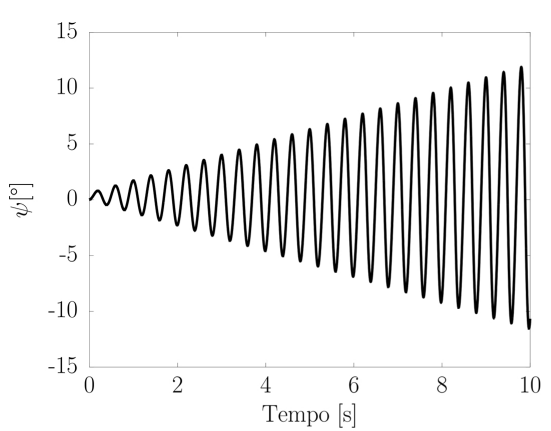

(a) $\psi(t)$.

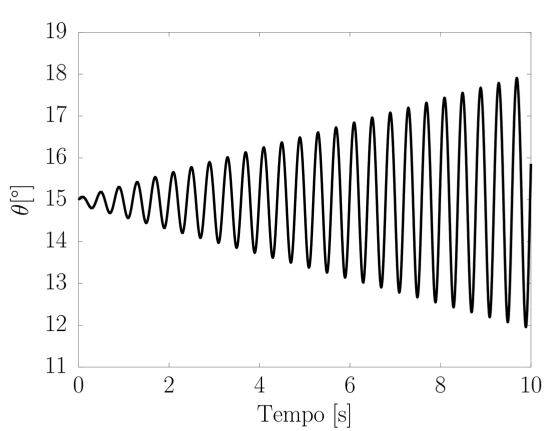

(b) $\theta(t)$.

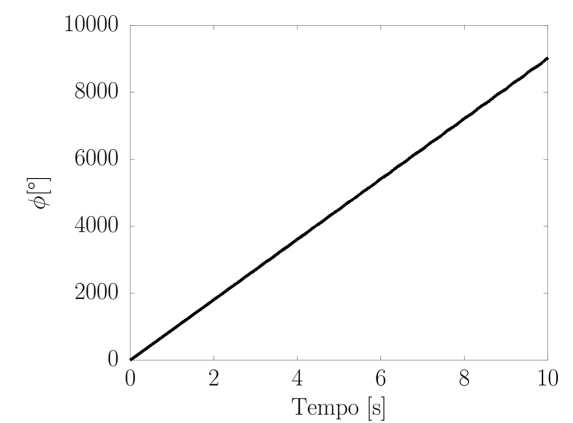

(c) $\phi(t)$.

Figura 3: Ângulos de Euler obtidos por cinemática inversa via ângulos de Euler para as condições iniciais: $\psi(0)=0.00^{\circ}$, $\dot{\psi}(0)=0.02[\mathrm{rad} / \mathrm{s}], \theta(0)=15.00^{\circ}, \dot{\theta}(0)=0.02[\mathrm{rad} / \mathrm{s}], \phi(0)=0.00^{\circ}$ e $\dot{\phi}(0)=15.7[\mathrm{rad} / \mathrm{s}]$.

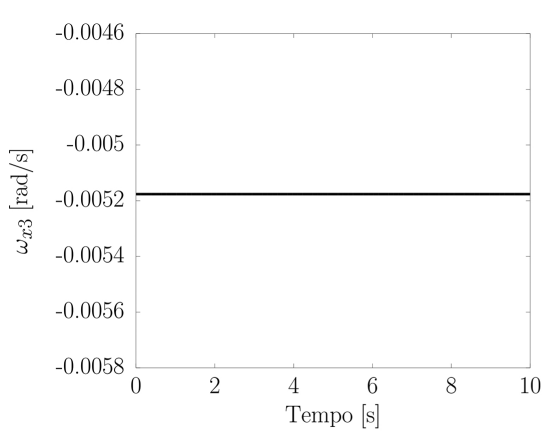

(a) $\omega_{x 3}$.

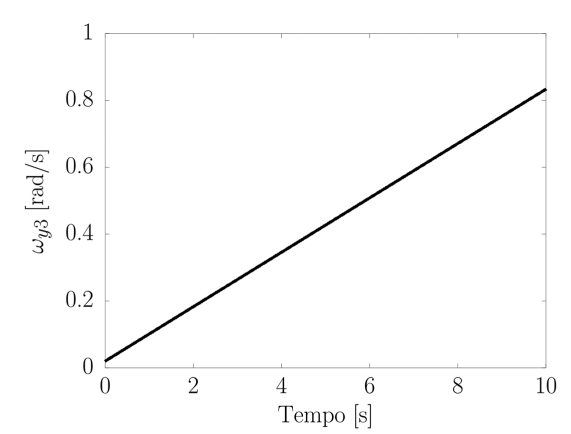

(b) $\omega_{y 3}$.

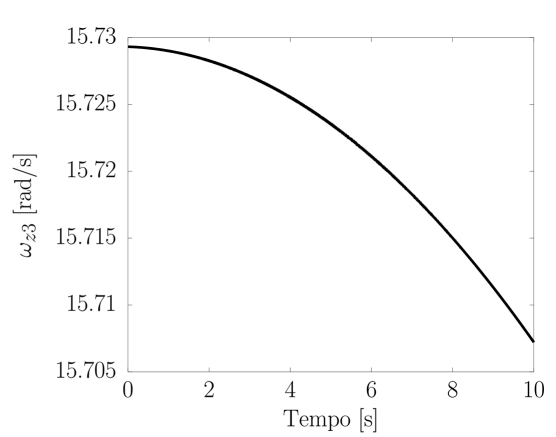

(c) $\omega_{z 3}$.

Figura 4: Velocidades angulares na direção principal de inércia $\omega_{x 3}, \omega_{y 3}$ e $\omega_{z 3}$ para as condições iniciais: $\psi(0)=0.00^{\circ}$, $\dot{\psi}(0)=0.02[\mathrm{rad} / \mathrm{s}], \theta(0)=15.00^{\circ}, \dot{\theta}(0)=0.02[\mathrm{rad} / \mathrm{s}], \phi(0)=0.00^{\circ}$ e $\phi(0)=15.7[\mathrm{rad} / \mathrm{s}]$. 


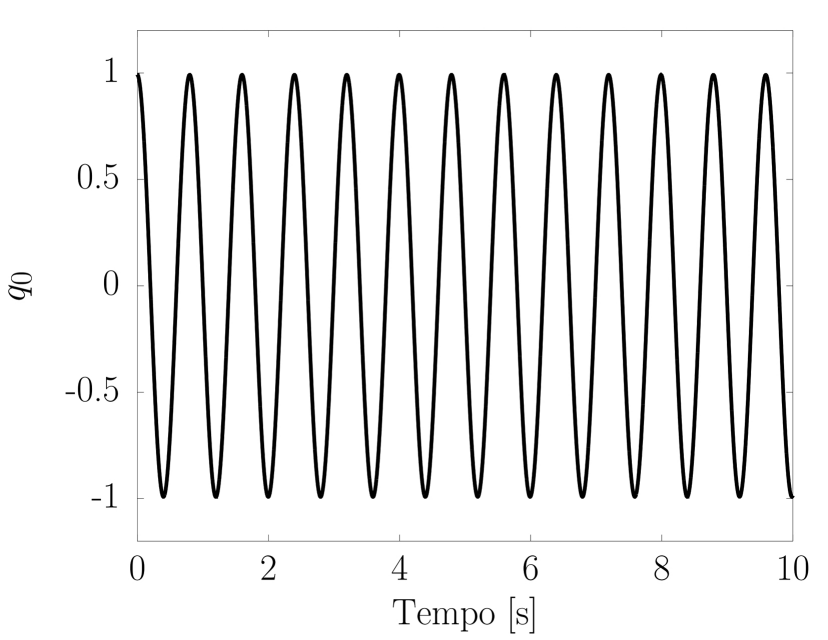

(a) $q_{0}$.

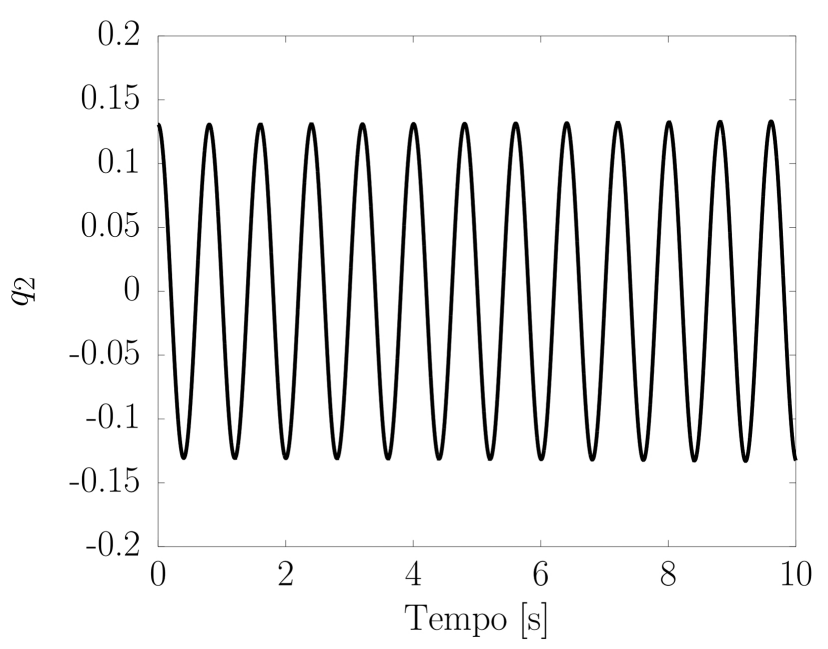

(c) $q_{2}$.

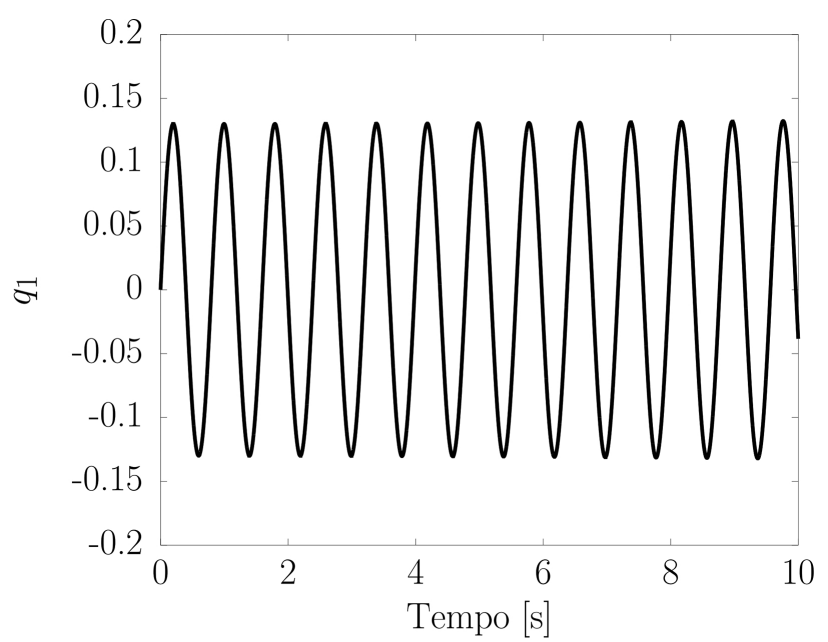

(b) $q_{1}$.

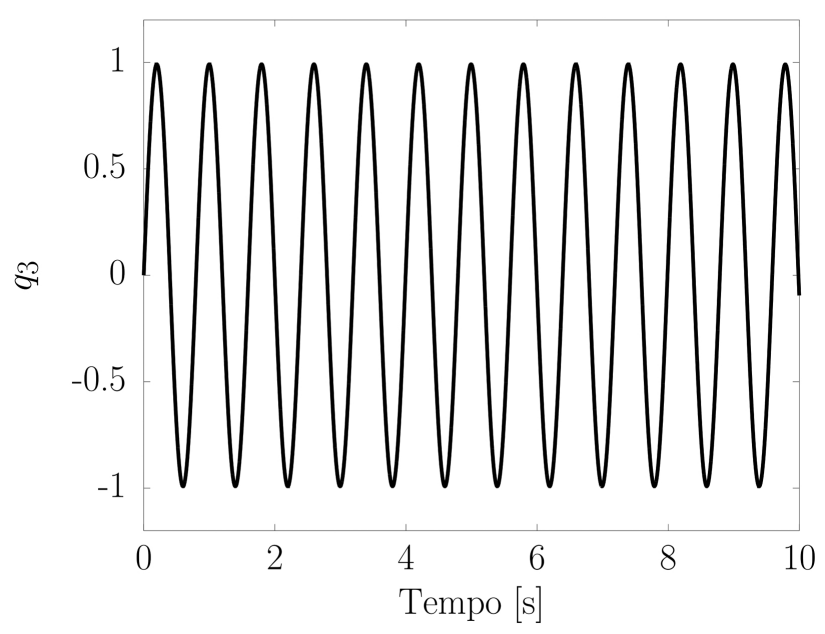

(d) $q_{3}$.

Figura 5: Componentes $q_{0}, q_{1}, q_{2}$ e $q_{3}$ do quatérnion $\hat{q}$, obtidas para as condições iniciais: $\psi(0)=0.00^{\circ}, \dot{\psi}(0)=0.02$ $[\mathrm{rad} / \mathrm{s}], \theta(0)=15.00^{\circ}, \dot{\theta}(0)=0.02[\mathrm{rad} / \mathrm{s}], \phi(0)=0.00^{\circ}$ e $\dot{\phi}(0)=15.7[\mathrm{rad} / \mathrm{s}]$.

Já para a solução do problema com singularidade, as figuras 6 e 7 expressam os resultados obtidos para os ângulos de precessão $\psi(t)$, nutação $\theta(t)$ e spin $\phi(t)$ pela cinemática inversa via velocidades angulares instantâneas e por quatérnions, respectivamente. Os resultados expressos nas figuras 6 e 7 deveriam ser idênticos, não fosse a influência das singularidades sob o resultado. A condição inicial do ângulo de nutação foi definida propositalmente com um valor para o qual $\operatorname{sen} \theta \approx 0$. A proximidade com um valor onde a eq. (19) é singular faz com que as soluções por meio da cinemática inversa por ângulos de Euler e por quatérnions apresentem resultados diferentes entre si. Não foi utilizado o valor exato $\theta=0$ devido aos resultados fornecidos serem numericamente indeterminados e impossibilitar a análise comparativa.

Para os ângulos de precessão $\psi(t)$ e spin $\phi(t)$, vistos nas figuras 6(a) e 6(c), correspondentes a solução por cinemática inversa através dos ângulos de Euler, é possível notar algumas mudanças bruscas nas trajetórias, enquanto na solução por quatérnions, figuras $7(\mathrm{a})$ e $7(\mathrm{c})$, tal comportamento não é percebido. O ângulo de nutação $\theta(t)$, fig. 6(c), também apresentou uma mudança repentina em seu comportamento que, na ausência de dissipação de energia ou momentos externos, pode ser facilmente atribuída às singularidades. 


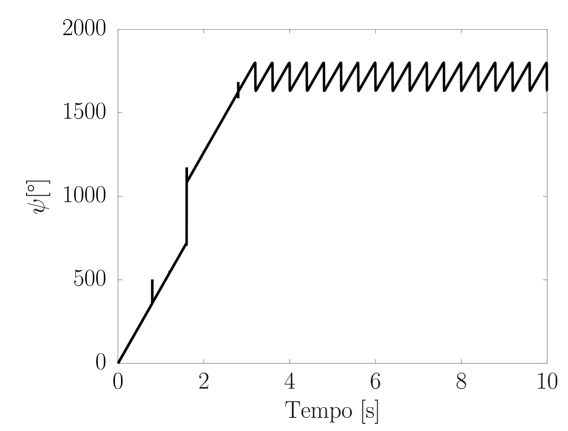

(a) $\psi(t)$.

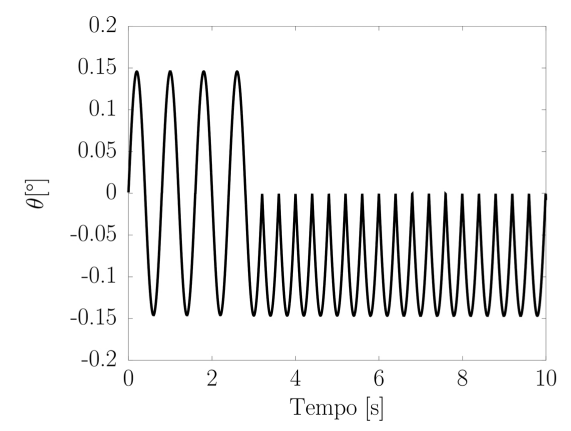

(b) $\theta(t)$.

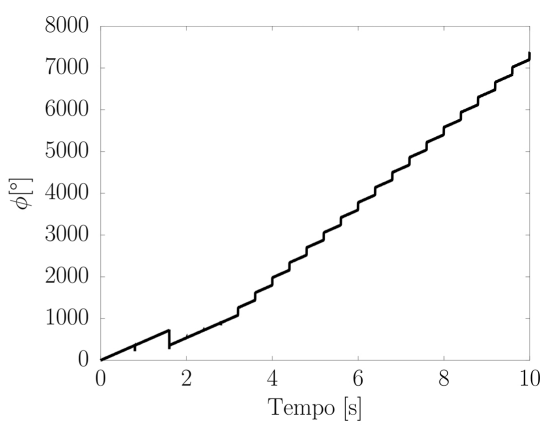

(c) $\phi(t)$.

Figura 6: Ângulos de Euler obtidos por cinemática inversa via velocidades angulares com as condições iniciais: $\psi(0)=0.00^{\circ}$, $\dot{\psi}(0)=0.02[\mathrm{rad} / \mathrm{s}], \theta(0)=0.001^{\circ}, \dot{\theta}(0)=0.02[\mathrm{rad} / \mathrm{s}], \phi(0)=0.00^{\circ}$ e $\dot{\phi}(0)=15.7[\mathrm{rad} / \mathrm{s}]$.

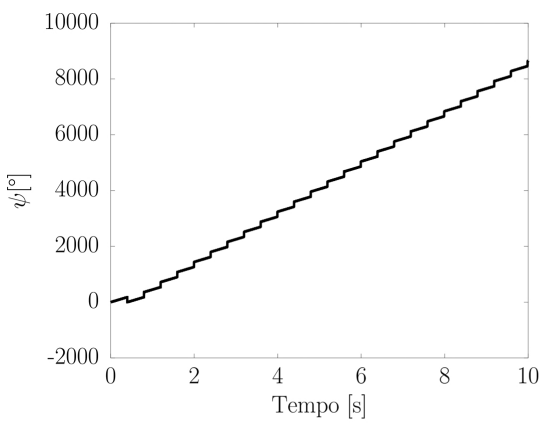

(a) $\psi(t)$.

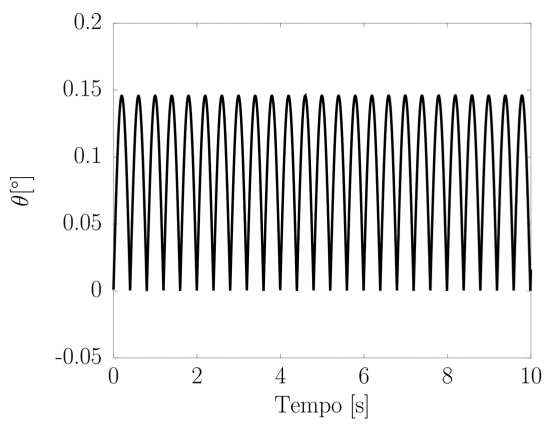

(b) $\theta(t)$.

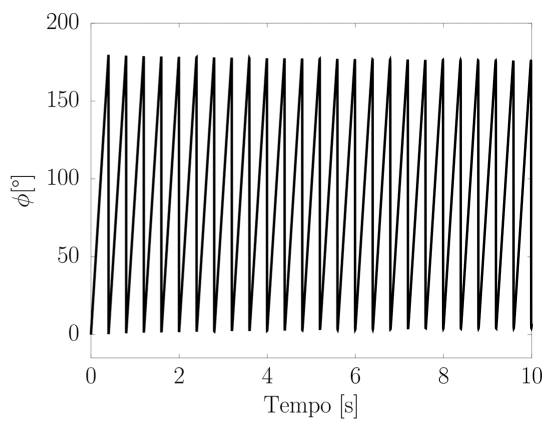

(c) $\phi(t)$.

Figura 7: Ângulos de Euler obtidos por cinemática inversa via quatérnions com as condições iniciais: $\psi(0)=0.00^{\circ}$, $\dot{\psi}(0)=0.02[\mathrm{rad} / \mathrm{s}], \theta(0)=0.001^{\circ}, \dot{\theta}(0)=0.02[\mathrm{rad} / \mathrm{s}], \phi(0)=0.00^{\circ}$ e $\dot{\phi}(0)=15.7[\mathrm{rad} / \mathrm{s}]$.

O efeito da singularidade no cálculo das velocidades de precessão $\dot{\psi}(t)$, nutação $\dot{\theta}(t)$ e spin $\dot{\phi}(t)$, realizado a partir da eq. (19) fica explícito na fig. 8 . onde nota-se que estas velocidades assumem valores muito altos em determinados instantes de tempo, o que ocorre a cada vez que o ângulo de nutação $\theta(t)$ se aproxima muito de $0^{\circ}$. Esse problema poderia ser parcialmente contornado escolhendo outra sequência de rotações para representar o giroscópio, porém as singularidades seriam apenas "transferidas" para outros valores de ângulos, já que obteríamos um sistema de equações semelhantes à eq. (19) com uma singularidade em $\cos \theta \approx 0$, ou seja, para $\theta=|n| \frac{\pi}{2}$, com $|n|=1,3,5 \ldots$, de modo que, utilizando ângulos de Euler para representar as rotações, nunca estaríamos de fato livres do problema. A escolha de outra sequência de rotações seria uma alternativa, por exemplo, na descrição de rotações de um corpo com baixa probabilidade de atingir determinados valores de ângulos por ação de alguma restrição imposta ao problema. Nestes casos, poderíamos admitir singularidades nessas faixas de valores improváveis para tais ângulos. Porém, para o caso onde o sis- tema pode assumir quaisquer valores de ângulos de nutação, a mudança na sequência das rotações não surtiria grande efeito.

Outra forma de evitar essas singularidades sem abrir mão do uso dos ângulos de Euler é discutido em [5], que propõe variar a sequência de rotações adotada de forma dinâmica, alterando a escolha da sequência de rotações entre as doze possíveis quando uma singularidade se aproxima para um determinado valor de ângulo, adotando uma sequência para à qual a singularidade seja deslocada para $\frac{\pi}{2}$ radianos de distância. Os autores apresentam ainda um algoritmo iterativo para a determinação da sequência de rotações ótima a ser adotada, que requer um número razoável de operações matemáticas. Frente à esta proposta, o uso de quatérnions apresenta um custo computacional muito menor e se mostra muito mais atraente para a solução do problema de cinemática inversa.

Na fig. 8(b) é possível notar um comportamento inconsistente para a velocidade de nutação, também gerado pela presença de singularidades. 


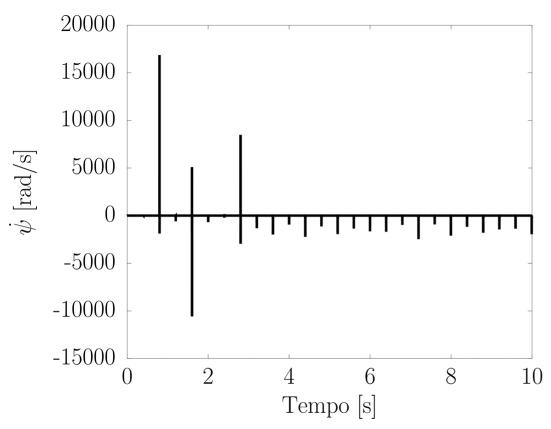

(a) $\dot{\psi}(t)$.

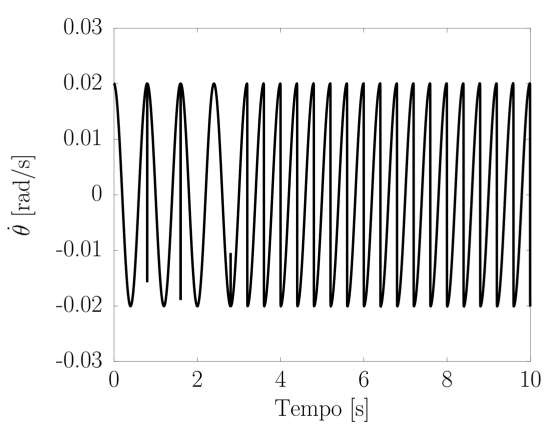

(b) $\dot{\theta}(t)$.

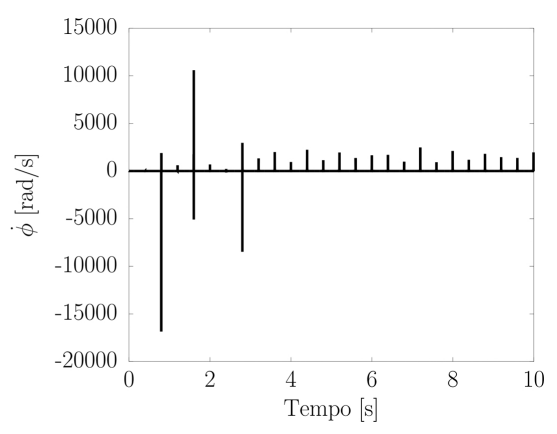

(c) $\dot{\phi}(t)$.

Figura 8: Velocidade de precessão $\dot{\psi}(t)$, nutação $\dot{\theta}(t)$ e spin $\dot{\phi}(t)$ obtidas por cinemática inversa via velocidades angulares com as condições iniciais: $\psi(0)=0.00^{\circ}, \dot{\psi}(0)=0.02[\mathrm{rad} / \mathrm{s}], \theta(0)=0.001^{\circ}, \dot{\theta}(0)=0.02[\mathrm{rad} / \mathrm{s}], \phi(0)=0.00^{\circ}$ e $\dot{\phi}(0)=15.7$ $[\mathrm{rad} / \mathrm{s}]$.

As singularidades não influenciam no cálculo das velocidades angulares nas direções principais de inércia, que são provenientes da integração da eq. (18), como pode ser visto na fig. 9 .

Já as componentes $q_{0}, q_{1}, q_{2}$ e $q_{3}$ do quatérnion $\hat{q}$ são apresentadas na fig. 10.

\section{Os Quatérnions Como uma Álgebra de Clifford}

Uma característica marcante dos quatérnions é o fato dos mesmos serem uma generalização do conjunto de números complexos $\mathbb{C}$. Esta ideia foi estendida e generalizada ainda mais com as ideias de Clifford, introduzidas em 1878, que permitiram trabalhar com elementos de dimensão bem maior. $\mathrm{Na}$ verdade, mesmo dimensões menores podem ser descritas álgebras de Clifford. Relações algébricas no espaço Euclidiano $\mathcal{V}$ podem ser descritas pelos elementos da bases $\mathbf{e}_{i}$ com as propriedades:

$$
\mathbf{e}_{i}^{2}=-1 \quad \mathbf{e}_{i} \mathbf{e}_{j}=-\mathbf{e}_{j} \mathbf{e}_{i} \quad \text { para } i \neq j
$$

Adicionando elementos da forma $\mathbf{e}_{i_{1}}, \cdots, \mathbf{e}_{i_{k}}$, sendo $i_{1}<\cdots<i_{k}$, e também dimensões $2^{n}$, sendo $n$ a dimensão do espaço $\mathcal{V}$, pode-se generalizar a álgebra de Clifford.

Tomando $\mathcal{V}$ um espaço vetorial no corpo dos reais $\mathbb{R}$ de dimensão $n$ com a aplicação bilinear $\mathcal{B}: \mathcal{V} \times \mathcal{V} \rightarrow$ $\mathbb{R}$, pode-se definir uma álgebra universal de Clifford 2 $C \ell(\mathcal{V}, \mathcal{B})$ com uma álgebra associativa gerada por $\mathcal{V}$, satisfazendo a relação fundamental:

$$
x^{2}=-\mathcal{B}(x, x)
$$

Sempre é possível escrever para $p+q=n$, sendo $p$ e $q$ o par de inteiros descrevendo a assinatura da forma quadrática:

$$
\mathcal{B}\left(\mathbf{e}_{i}, \mathbf{e}_{i}\right)=\left\{\begin{array}{cc}
1, & i=1,2, \ldots, p \\
-1, & i=1,2, \ldots, q
\end{array}\right.
$$

sendo uma álgebra de Clifford $C \ell(\mathcal{V}, \mathcal{B})$ nomeada de $C \ell_{p, q}$ e formada pelos elementos ortogonais entre

\footnotetext{
${ }^{2}$ Alguns autores apontam que quando se trabalha no corpo dos reais a álgebra é dita geométrica.
}

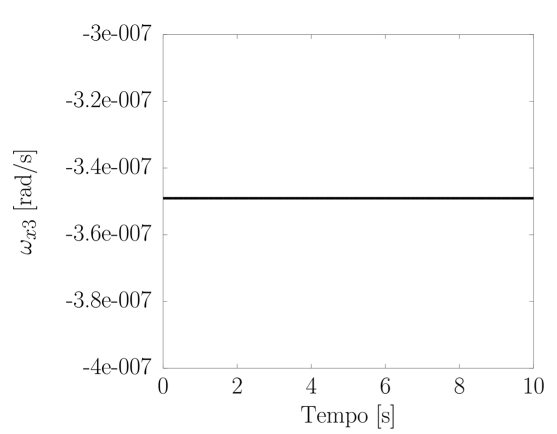

(a) $\omega_{x 3}$.

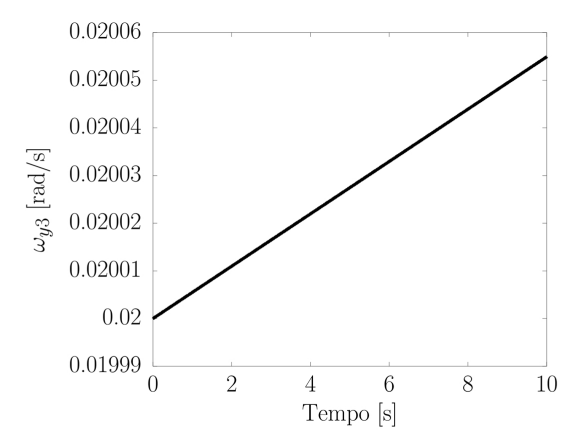

(b) $\omega_{y 3}$.

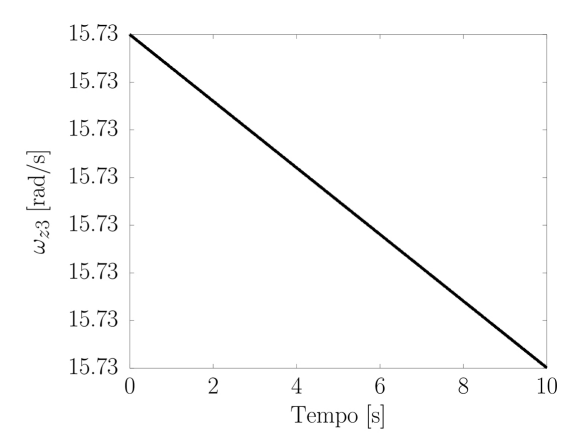

(c) $\omega_{z 3}$.

Figura 9: Velocidades angulares na direção principal de inércia $\omega_{x 3}, \omega_{y 3}$ e $\omega_{z 3}$ com as condições iniciais: $\psi(0)=0.00^{\circ}$, $\dot{\psi}(0)=0.02[\mathrm{rad} / \mathrm{s}], \theta(0)=0.001^{\circ}, \dot{\theta}(0)=0.02[\mathrm{rad} / \mathrm{s}], \phi(0)=0.00^{\circ}$ e $\phi(0)=15.7[\mathrm{rad} / \mathrm{s}]$. 


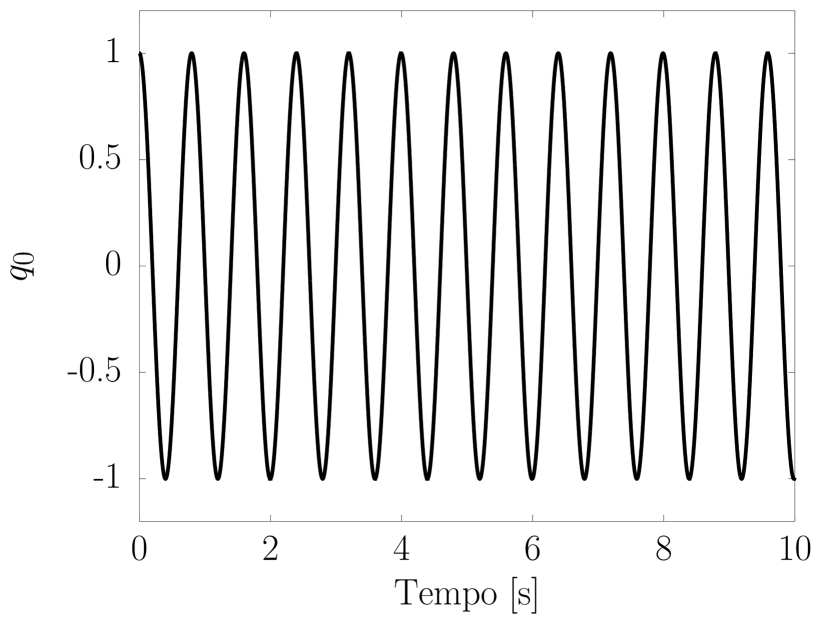

(a) $q_{0}$.

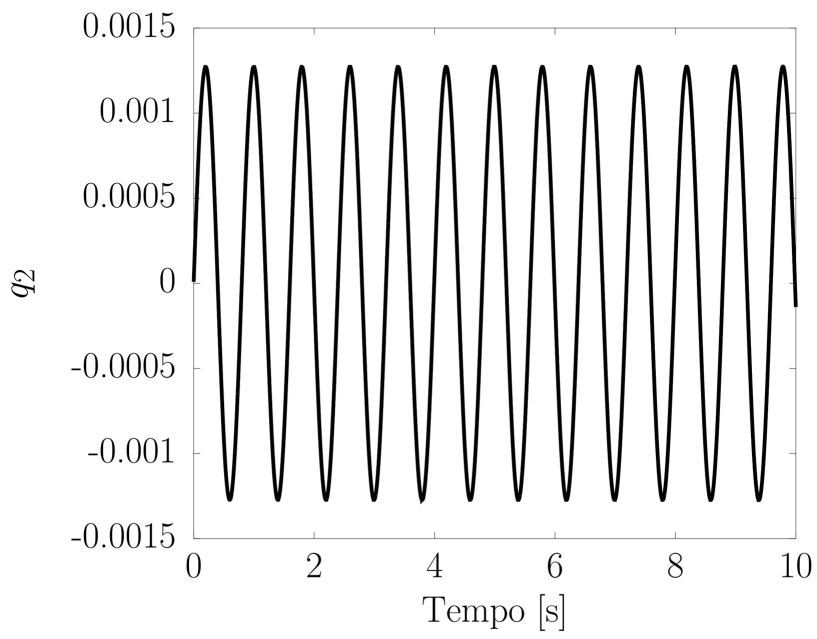

(c) $q_{2}$.

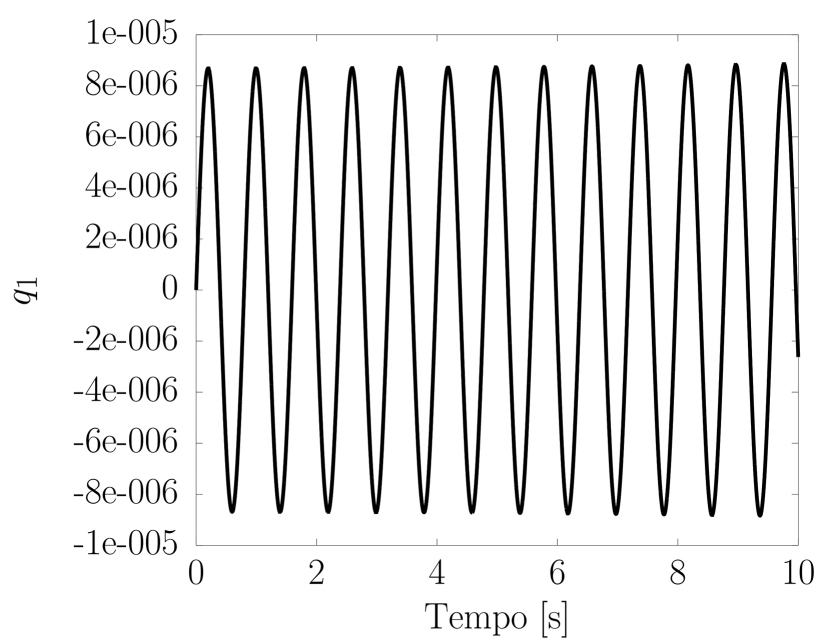

(b) $q_{1}$.

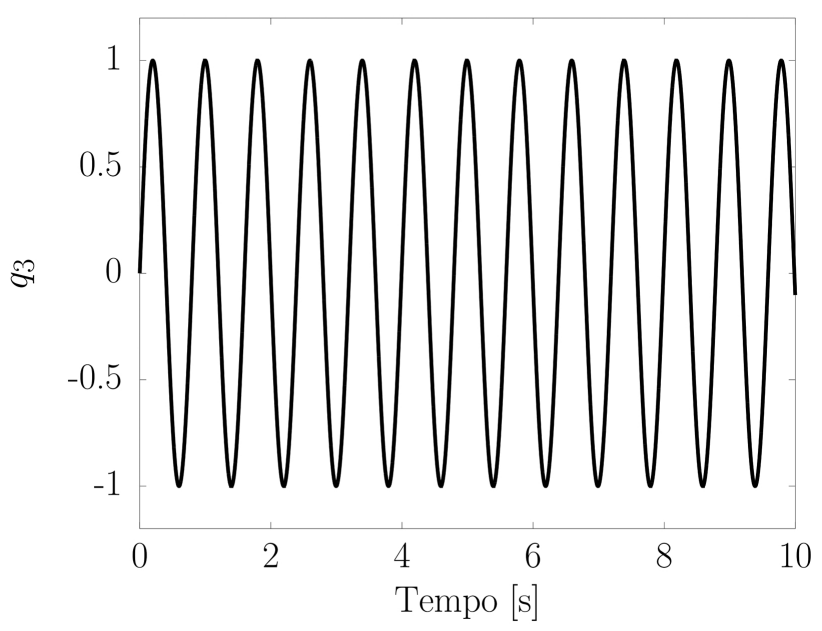

(d) $q_{3}$.

Figura 10: Componentes $q_{0}, q_{1}, q_{2}$ e $q_{3}$ do quatérnion $\hat{q}$, obtidas para as condições iniciais: $\psi(0)=0.00^{\circ}, \dot{\psi}(0)=0.02$ $[\mathrm{rad} / \mathrm{s}], \theta(0)=0.001^{\circ}, \dot{\theta}(0)=0.02[\mathrm{rad} / \mathrm{s}], \phi(0)=0.00^{\circ}$ e $\dot{\phi}(0)=15.7[\mathrm{rad} / \mathrm{s}]$.

si, com os $p$ elementos com norma 1 e $q$ elementos com norma -1 :

$$
x=\sum_{A} x_{A} \mathbf{e}_{A}
$$

sendo $x_{A}$ números reais e :

$$
A=\left\{i_{1}, i_{2}, \cdots, i_{k} \mid \quad 1 \leq i_{1} \leq i_{2} \leq \cdots \leq i_{k} \leq n\right\}
$$

e

$$
\mathbf{e}_{A}=\mathbf{e}_{1} \mathbf{e}_{2} \cdots \mathbf{e}_{i_{k}}, \quad \mathbf{e}_{\oslash}=\mathbf{e}_{0}=1
$$

Assim, pode-se constatar que a álgebra dos reais $\mathbb{R}$ quando $n=0$ é uma álgebra de Clifford $C \ell_{0,0} \simeq \mathbb{R} ;$ já quando $n=1, C \ell_{0,1} \simeq \mathbb{C}$ e quando $n=2, C \ell_{0,2} \simeq \mathbb{H}$, mostrando que os quatérnions são uma álgebra de Clifford formada por escalares, vetores e bivetores.

Considerando $\mathcal{V} \simeq \mathbb{R}^{2}$ o espaço vetorial gerado por $\mathbf{e}_{1}$ e $\mathbf{e}_{2}$, a forma de um elemento qualquer nesta álgebra $C \ell_{0,2}$ será descrita por:

$$
\hat{q}=q_{0}+q_{1} \mathbf{e}_{1}+q_{2} \mathbf{e}_{2}+q_{3} \mathbf{e}_{1} \mathbf{e}_{2}
$$

Interessante observar que, se for tomado $i=\mathbf{e}_{1}$, $j=\mathbf{e}_{2}$ e $k=\mathbf{e}_{1} \mathbf{e}_{2}$, tem-se a relação fundamental da álgebra dos quatérnions $i^{2}=j^{2}=k^{2}=i j k=-1$, já descrita anteriormente, e o quatérnion $\hat{q}$, exatamente da forma da eq. (2).

Uma excelente introdução neste tema, acessível em língua portuguesa, é o livro de Jayme Vaz e Roldão da Rocha [37. 


\section{Conclusões}

Este artigo exemplificou, a partir de uma aplicação com um giroscópio, como os quatérnions são úteis e interessantes na descrição de rotações e na solução de problemas de cinemática inversa, com diversas vantagens frente a outras opções de parametrização de rotações. Porém, além destes trunfos numéricos, os quatérnions são elementos importantes por formarem a primeira álgebra não-comutativa da história e servirem de porta de entrada para outros sistemas algébricos mais interessantes para descrição matemática de diversos sistemas físicos. Infelizmente, o tema apresentado neste artigo não é, por via de regra, seguido nos cursos de mecânica, tanto para físicos como para engenheiros.

Os autores acreditam ser necessário abordar mais aspectos sobre parametrização de rotação e apresentar os quatérnions não apenas em cursos de mecânica, mas também em disciplinas de álgebra linear e/ou matemática aplicada. Assim, os autores fazem frente aos que defendem a necessidade de um curso de álgebra geométrica no ensino de física, matemática e em cursos de engenharia.

\section{Agradecimentos}

O primeiro autor agradece a CAPES por sua bolsa de mestrado. Os autores também agradecem o apoio financeiro do CNPq e da FEPISA, além dos comentários e sugestões do editor e dos revisores da RBEF.

\section{Referências}

[1] L. Euler, Novi Acad. Sci. Petrop 20, 189 (1775).

[2] J. Stuelpnagel, SIAM review 6, 422 (1964).

[3] J. Diebel, Matrix 58, 15 (2006).

[4] J. Neto, Mecânica Newtoniana, Lgrangiana e Hamiltoniana (Editora Livraria da Física, São Paulo, 2004).

[5] P. Singla, D. Mortari and J.L. Junkins, in: Proceedings of the AAS Space Flight Mechanics Conference, Hawaii, 2004.

[6] P. Betsch, A. Menzel and E. Stein, Computer Methods in Applied Mechanics and Engineering 155, 273 (1998).

[7] M. Trindade and R. Sampaio, Journal of the Brazilian Society of Mechanical Sciences 22, 341 (2000).

[8] J. Kuipers, Quaternions and Rotation Sequences: A Primer with Applications to Orbits, Aerospace, and Virtual Reality (Princeton Paperbacks, Princeton University Press, 1999).
[9] K. Vogtmann, V. Arnold and A. Weinstein, Mathematical Methods of Classical Mechanics (Graduate Texts in Mathematics) (Springer, New York, 1989).

[10] H. Goldstein, C. Poole and J. Safko, Classical Mechanics (Addison Wesley, New York, 2002).

[11] L. Meirovitch, Methods of Analytical Dynamics, Dover Civil and Mechanical Engineering (Dover Publications, Mineola, 2012).

[12] E.T. Whittaker, A Treatise on the Analytical Dynamics of Particles and Rigid Bodies: With an Introduction to the Problem of Three Bodies (CPU Archive, London, 1970).

[13] W.R. Hamilton, Elements of Quaternions (Longmans, Green, \& Company, Londres, 1866).

[14] W.R. Hamilton, The Mathematical Papers of Sir William Rowan Hamilton, (CUP Archive, London, 2000).

[15] W.R. Hamilton, Lectures on Quaternions, (The Royal Irish Academy, Dublin, 1853).

[16] M. Menon, Revista Brasileira de Ensino de Física 31, 2305 (2009).

[17] Gustavo Martínez-Sierra y Pierre Francois Benoit Poirier, Latin-American Journal of Physics Education 2, 201 (2008).

[18] M.J. Amoruso, Euler Angles and Quaternions in Six Degree of Freedom Simulations of Projectiles Technical Report (DTIC Document, Fort Belvoir, 1996).

[19] F. Lizarralde and J.T. Wen, IEEE Transactions on Automatic Control 41, 468 (1996).

[20] M.D. Shuster, Navigation 8, 439 (1993).

[21] K. Shoemake, Quaternion Calculus and Fast Animation, Computer Animation: 3-D Motion Specification and Control (Siggraph, Anaheim, 1987).

[22] J.C. Hart, G.K. Francis and L.H. Kauffman, ACM Transactions on Graphics 13, 256 (1994).

[23] W.R. Rudnicki, G. Bakalarski and B. Lesyng, Journal of Biomolecular Structure and Dynamics 17, 1097 (2000).

[24] G. Harauz, Ultramicroscopy 33, 209 (1990).

[25] T. Róg, K. Murzyn, K. Hinsen and G.R. Kneller, Journal of Computational Chemistry 24, 657 (2003).

[26] W. Sprössig, Advances in Applied Clifford Algebras 17, 259 (2007).

[27] J.D. Gibbon, D.D. Holm, R.M. Kerr and I. Roulstone, Nonlinearity 19, 1969 (2006).

[28] H. Eshraghi and J. Gibbon, Journal of Physics A: Mathematical and Theoretical 41, 344004 (2008).

[29] J. Vaz Jr., Revista Brasileira de Ensino de Física 19, 234 (1997).

[30] D.H.Z. Carrera, Movimento de Rotação Sem Restrição de um Corpo Rígido. Tese de Doutorado, PUC, Rio de Janeiro, 2010.

[31] A.A.G. Oliveira, Estudo Estatístico dos Processos Envolvidos em uma Plataforma de Atitude Solidária. Dissertação de Mestrado, Instituto Nacional de Pesquisas Espaciais, São José dos Campos, 2000. 
[32] S.L. Altmann, Mathematics Magazine 62, 291 (1989).

[33] R.S. Lima and M.P. Mello, CNMAC - Congresso Nacional de Matemática Aplicada e Computacional (2004).

[34] V. Pisacane, Fundamentals of Space Systems (Oxford University Press, Oxford, 2005).

[35] R.A. Wehage, in: Computer Aided Analysis and Optimization of Mechanical System Dynamics (Springer, Berlin, 1984), p. 147-180.

[36] R.A. Mayo, Journal of Guidance, Control, and Dynamics 2, 44 (1979).

[37] J. Vaz and R. Da Rocha Jr., Álgebras de Clifford e Espinores (Livraria da Física, São Paulo, 2012). 